\title{
Case Report: Multidrug-Resistant Mycobacterium leprae in a Case of Smear-Negative Relapse
}

\author{
Divya Kamat, ${ }^{1}$ Tarun Narang, ${ }^{1 *}$ Madhvi Ahuja, ${ }^{2}$ Mallika Lavania, ${ }^{2}$ and Sunil Dogra ${ }^{1}$ \\ ${ }^{1}$ Department of Dermatology, Venereology and Leprology, Postgraduate Institute of Medical Education and Research, Chandigarh, India; \\ ${ }^{2}$ Department of Molecular Biology, Stanley Browne Laboratory, TLM Community Hospital, New Delhi, India
}

\begin{abstract}
The ongoing transmission of leprosy in India is worrisome, and emerging drug resistance may be one of the factors responsible for the continued transmission of leprosy in India. Emerging cases of multidrug-resistant Mycobacterium leprae pose a great threat to eradication of leprosy and must be addressed with utmost priority. We report a case of multidrug-resistant $M$. leprae in a case of relapse where slit skin smear (SSS) was negative and histopathology was inconclusive. Drug resistance studies in leprosy are undertaken only in smear-positive relapse cases, and detection of this type of multidrug resistance in a case with negative SSS and innocuous histopathology is rather unusual and highlights the importance of undertaking drug resistance tests even in smear-negative cases of leprosy relapse. Resistance to ofloxacin (OFL) is also a cause for concern as OFL is one of the reserve drugs recommended for treatment of rifampicin-resistant strains.
\end{abstract}

\section{INTRODUCTION}

Leprosy continues to be endemic in parts of India and 60\% of new cases worldwide are reported from India alone. Despite a declining prevalence rate, the annual new case detection rate has remained stable around 9.27 per lakh population over the last decade. ${ }^{1}$ The ongoing transmission of leprosy in India is alarming, and various reasons have been proposed. One of the reasons could be emerging drug resistance to currently used drugs in WHO multidrug therapy (MDT). Drug resistance in Mycobacterium leprae to dapsone was earliest reported in 1964; subsequently, there were reports of resistance to rifampicin (RMP) in 1976 and to ofloxacin (OFL) in $1996 .{ }^{2}$ Emerging cases of multidrug-resistant $M$. leprae ${ }^{2}$ are a threat to our dream of zero leprosy and must be addressed with utmost priority. We report a case of multidrug-resistant $M$. leprae in a case of relapse where slit skin smear (SSS) was negative and histopathology was inconclusive.

Report of a case. A 35-year-old man who was diagnosed with leprosy 3 years ago and treated with 12 months of multibacillary multidrug therapy presented with reappearance of multiple hypopigmented patches all over the body since 4 months and shooting pain in forearms along the distribution of ulnar nerve for the last 1 month. The baseline spectrum of leprosy, bacteriological index, and other details were unknown as he had lost his past medical records, but he informed that most of his lesions had resolved after completion of the treatment. On mucocutaneous examination, about 25 symmetrically distributed hypopigmented and hypoesthetic plaques were present all over the body (Figure 1). There was no erythema, edema, or tenderness of the lesions to suggest type 1 reaction. He also had symmetrical thickening of the ulnar nerves, radial cutaneous nerves, common peroneal nerves, and sural nerves along with tenderness of both ulnar nerves. There was no glove-and-stocking anesthesia or motor weakness. Slit skin smears from ear lobes and patches were negative. Histopathology of skin biopsy performed from the hypopigmented patch over the back revealed mild lymphomononuclear infiltrate with preserved adnexal structures and

\footnotetext{
* Address correspondence to Tarun Narang, Department of Dermatology, Venereology and Leprology, Nehru Hospital, Post Graduate Institute of Medical Education and Research, Sector 12, Chandigarh,
} India, 160012. E-mail: narangtarun@yahoo.co.in absence of granulomas or foamy histiocytes. Fite stain for lepra bacilli was negative. In view of new onset of skin lesions and neuritis after 2 years of completion of MDT, we considered a diagnosis of relapse with bilateral ulnar neuritis even though he did not strictly fulfill the WHO criteria for relapse. WHO MBR MDT was re-initiated along with tablet prednisolone $40 \mathrm{mg}$ daily for management of recent-onset neuritis. Prednisolone was gradually tapered over 16 weeks with improvement in neuritis. However, the patient continued to develop new hypopigmented patches with progression of the preexisting patches. A skin biopsy specimen from the forearm for drug resistance studies revealed resistance to both RMP and OFL. DNA was extracted from the biopsy specimen by using the Qiagen Blood and Tissue DNA Extraction Kit (Qiagen, Germantown, MD). Quantitative polymerase chain reaction (qPCR)-high-resolution melting (HRM) analysis for drug susceptibility testing of $M$. leprae was performed for RMP- and OFL-resistant $M$. leprae (Figure 2). Conventional polymerase chain reaction (PCR) was performed as per the WHO protocol for all three drugs RMP, dapsone, and OFL targeting $r p o B$, folP, and gyrA genes of $M$. leprae, respectively. DNA sequencing and HRM analysis of the patient sample revealed mutations detected at codon positions T433I and D441Y for RMP and G89C for OFL (Figure 3). He was then initiated on second-line anti-leprosy therapy (minocycline $100 \mathrm{mg}$, clarithromycin $500 \mathrm{mg}$, and clofazimine $50 \mathrm{mg}$-once daily each). The patient tolerated the alternate leprosy regimen well and after 4 months of this regimen, and the patient reported improvement of the preexisting lesions and neuritis.

\section{DISCUSSION}

The first case of multidrug-resistant $M$. leprae (resistance to dapsone, RMP, and OFL) was reported from Mali in 1997. ${ }^{3}$ The recent global WHO antimicrobial surveillance in leprosy reported an overall resistance rate of $8.0 \%$ (total number of cases 1932) among new and relapse cases from 19 endemic countries. Multidrug resistance was found in 24 strains; however, none of the strains were resistant to both RMP and OFL or to all three drugs. All these strains of multidrug resistance were reported from India, Brazil, and Indonesia. ${ }^{4}$ In a multicenter study from Japan, Indonesia, Pakistan, and Haiti, the rate of multidrug resistance was found to be $14.8 \%$ with two strains showing resistance to all three drugs. ${ }^{5}$ In a study 

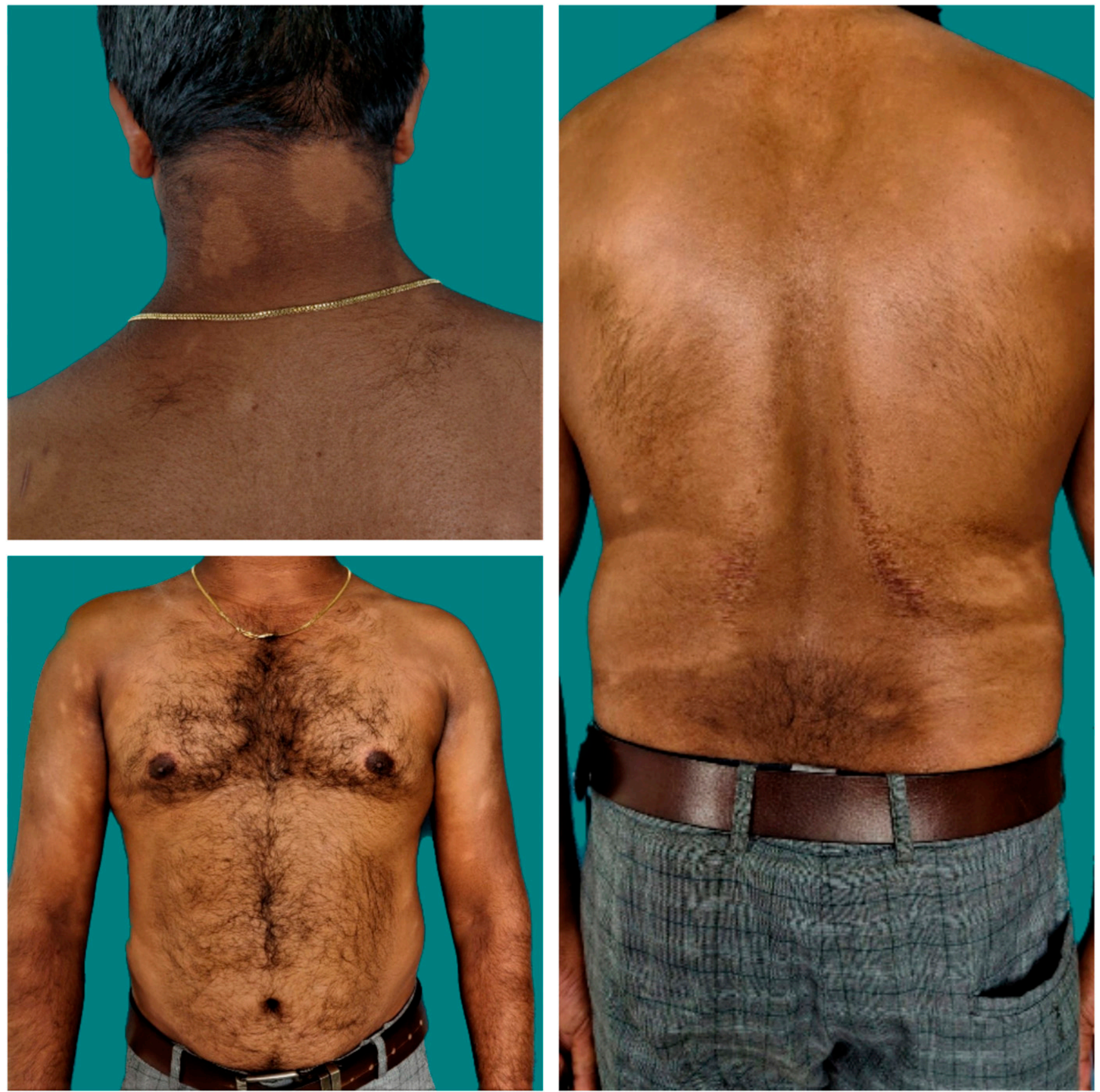

FIGURE 1. Multiple symmetrically distributed hypopigmented, hypoasthetic patches over the trunk, upper extremities, and nape of the neck. This figure appears in color at www.ajtmh.org.

from India, multidrug resistance among 239 relapse cases was studied. Multidrug resistance to any two drugs was found in 15 strains (seven-dapsone and RMP, seven-dapsone and OFL, and one-RMP and OFL), and two strains showed resistance to all three drugs. ${ }^{6}$

Molecular drug susceptibility assays for $M$. leprae are based on PCR amplification and detection of mutant alleles in the drug resistance-determining regions of folP1, rpoB, and gyrA for dapsone, RMP, and OFL, respectively. Among these assays, PCR followed by DNA sequencing is currently the "molecular gold standard." However, considering the high cost of this method, qPCR-HRM has been increasingly used as a screening tool in endemic regions with $100 \%$ specificity for detection of mutations in drug-resistant M. leprae. ${ }^{7}$

Untreated cases of multibacillary leprosy are a large reservoir of bacilli, and it is possible that a single patient can harbor thousands of bacilli with resistance to more than one drug. The drug-resistant subpopulation of bacilli increases because of inadequate therapy or noncompliance to the prescribed treatment, and this not only makes the treatment of that individual extremely challenging but also poses a serious threat to the ultimate goal of leprosy eradication due to transmission of drug-resistant strains in the community. ${ }^{2}$ Familial transmission of primary multidrug resistance (to RMP and dapsone) 

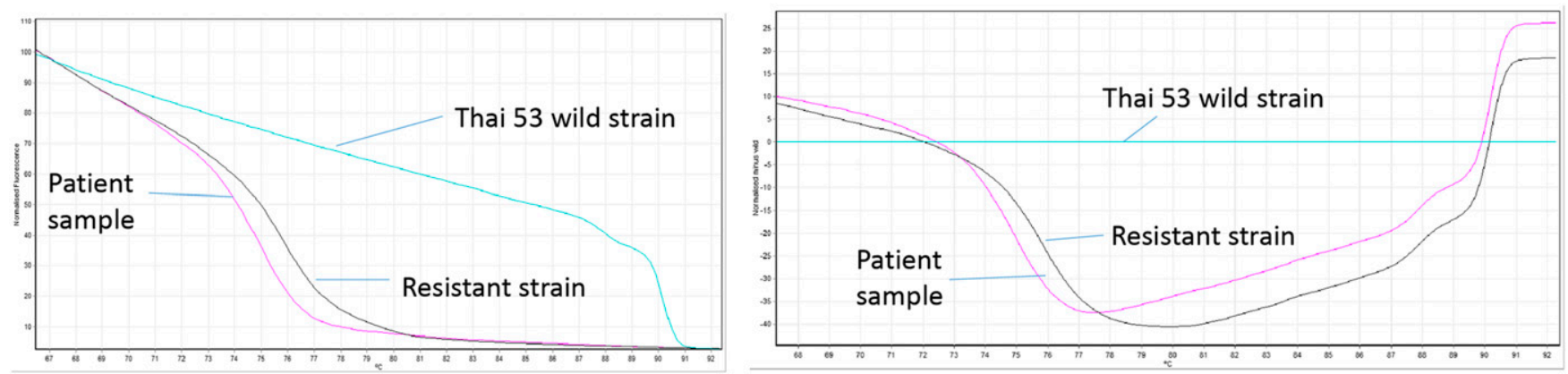

FIGURE 2. qPCR-high resolution melting (HRM), drug susceptibility testing of Mycobacterium leprae results of rifampicin-resistant $M$. leprae-normalized and difference plots of graphic display of the post-qPCR HRM analysis of the rpo $B$ drug resistance-determining region of $M$. leprae. DNA melting curves obtained in the analysis of mutant strains deviate from the wild-type profile (Thai-53 strain, shown in blue color). This figure appears in color at www.ajtmh.org.

was highlighted in a study from a hyperendemic region in Brazil. $^{8}$ Drug-resistant cases can also clinically present as steroid nonresponsive type 1 reaction $^{9}$ or as chronic steroiddependent type 2 reaction, ${ }^{10}$ which if left undetected can further increase the burden of disability.

Multidrug resistance to two bactericidal drugs in a case of relapse as seen in the index patient is a cause of grave concern. Normally, drug resistance is suspected in smearpositive cases of relapse and detection of this type of multidrug resistance in a case with negative SSS and innocuous histopathology is rather unusual and highlights the importance of undertaking drug resistance testing in all cases of relapse; even in smear-negative cases. Moreover, resistance to OFL is also an alarming issue as it is one of the reserve drugs recommended for RMP-resistant strains. In the last 2-3 decades, there has not been much research on new drugs which are effective against $M$. leprae, and if the current scenario worsens or progresses, we will not be left with options to treat multidrug-resistant leprosy cases and we may be heading toward the same fate as tuberculosis, where extensively drug-resistant TB and total drug resistance are being seen.

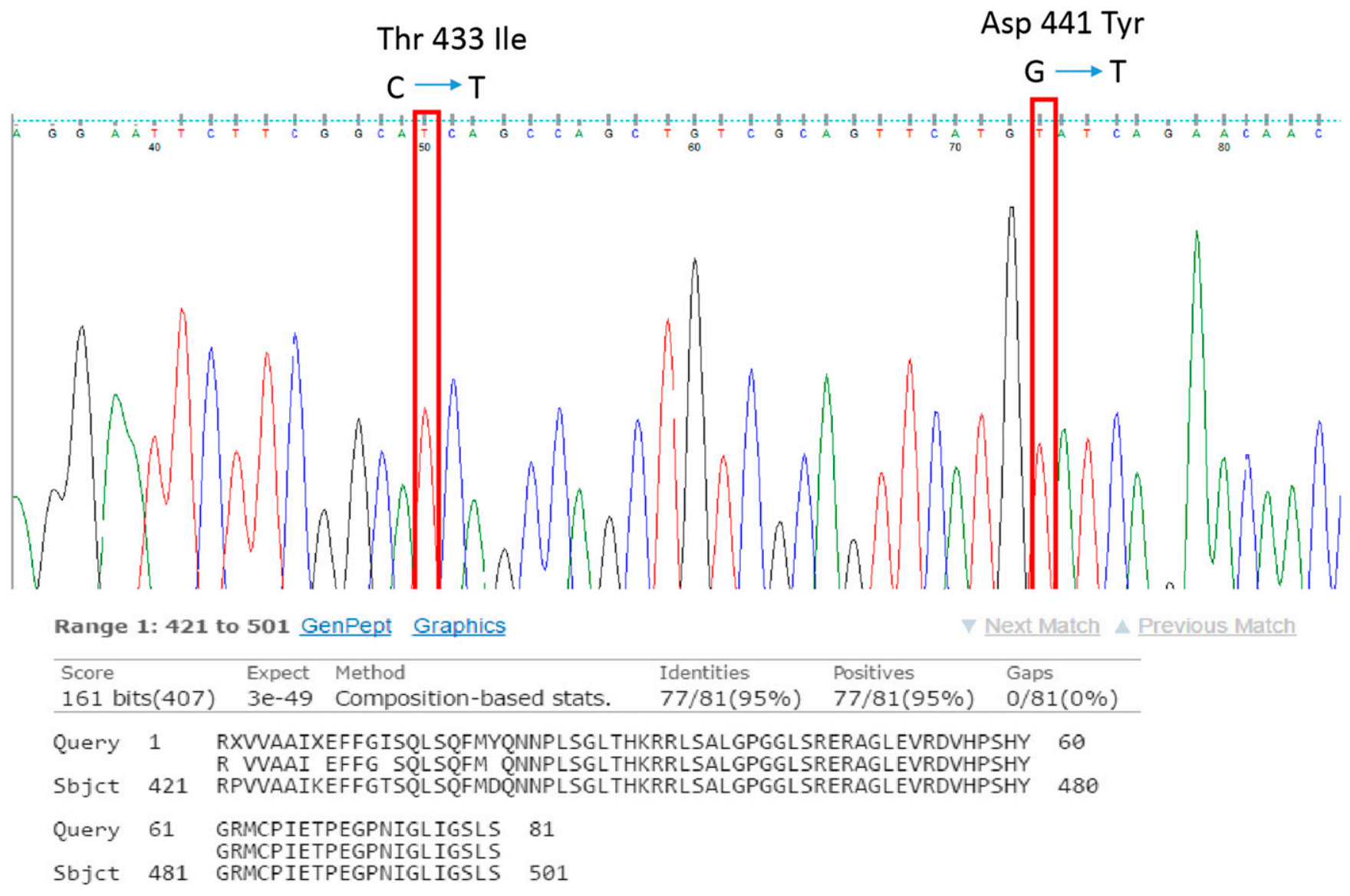

FIGURE 3. DNA chromatogram result for the DNA sequence of $r p o B$ drug resistance-determining region of Mycobacterium leprae sample, showing two independent mutations associated with RMP resistance. This figure appears in color at www.ajtmh.org. 
Received December 6, 2019. Accepted for publication January 8, 2020.

Published online February 10, 2020.

Acknowledgment: The American Society of Tropical Medicine and Hygiene (ASTMH) assisted with publication expenses.

Authors' addresses: Divya Kamat, Tarun Narang, and Sunil Dogra, Department of Dermatology, Venereology and Leprology, Postgraduate Institute of Medical Education and Research, Chandigarh, India, E-mails: divyakamat27@gmail.com, narangtarun@yahoo.co.in, and sundogra@hotmail.com. Madhvi Ahuja and Mallika Lavania, Department of Molecular Biology, Stanley Browne Laboratory, TLM Community Hospital, New Delhi, India, E-mails: madhviahuja87@ gmail.com and mallikalavania@gmail.com.

\section{REFERENCES}

1. Government of India, 2018. National Leprosy Eradication Programme. Available at: http://www.nlep.nic.in/data.html.

2. Williams DL, Gillis TP, 2012. Drug-resistant leprosy: monitoring and current status. Lepr Rev 83: 269.

3. Cambau E, Perani E, Guillemin I, Jamet P, Ji B, 1997. Multidrugresistance to dapsone, rifampicin, and ofloxacin in Mycobacterium leprae. Lancet 349: 103-104.
4. Cambau E et al., 2018. Antimicrobial resistance in leprosy: results of the first prospective open survey conducted by a WHO surveillance network for the period 2009-15. Clin Microbiol Infect 24: 1305-1310.

5. Maeda S, Matsuoka M, Nakata N, Kai M, Maeda Y, Hashimoto K, Kimura H, Kobayashi K, Kashiwabara Y, 2001. Multidrug resistant Mycobacterium leprae from patients with leprosy. Antimicrob Agents Chemother 45: 3635-3639.

6. Lavania M et al., 2018. Molecular detection of multidrug-resistant Mycobacterium leprae from Indian leprosy patients. J Glob Antimicrob Resist 12: 214-219.

7. Araujo S et al., 2017. qPCR-High resolution melt analysis for drug susceptibility testing of Mycobacterium leprae directly from clinical specimens of leprosy patients. PLoS Negl Trop Dis 11: e0005506.

8. Rosa PS et al., 2019. Emergence and transmission of drug-/ multidrug-resistant Mycobacterium leprae in a former leprosy colony in the Brazilian amazon. Clin Infect Dis: ciz570.

9. SinhaS, Sardana K, Agrawal D, Malhotra P, Lavania M, Ahuja M, 2019. Multidrug resistance as a cause of steroid-nonresponsive downgrading type I reaction in Hansen's disease. Int J Mycobacteriol 8: 305-308.

10. Arora P, Sardana K, Agarwal A, Lavania M, 2019. Resistance as a cause for chronic steroid dependent ENL - a novel paradigm with potential implications in management. Lepr Rev 90: 201-205. 